Chapter 4

\title{
Propagation of Electromagnetic Waves in Anisotropic Photonic Structures
}

\author{
V.I. Fesenko, I.A. Sukhoivanov, S.N. Shul'ga and \\ J.A. Andrade Lucio
}

Additional information is available at the end of the chapter

http://dx.doi.org/10.5772/54847

\section{Introduction}

In this chapter we discuss plane-wave propagation in a layered arbitrarily anisotropic media.One-dimensional (1D) arbitrary layered structure is usually formed by stacking together layers of several different materials with some specific thickness $d_{j}$ and refractive index $n_{j}$ as depicted on Fig.1.

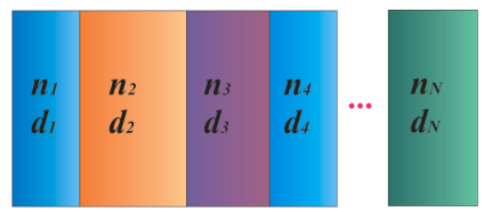

Figure 1. Arbitrary layered structure

Nowadays layered photonic structures (LPSs) are key of optoelectronic and microwave devices such as, phased-array antennas, microcavities and mirrors [1,2], filters of xWDM systems [3], waveguide structures, photodetectors, sensors and others. In case of active devices the layered structures are usually used in form of superlattices [4-6], multiple quantum wells $[7,8]$ and asymmetric multiple quantum wells [9].

Different kinds of materials are used today for design LPSs, such as linear and nonlinear $[3,10]$ dielectric materials, anisotropic or bi-anisotropic materials [11], chiral media [12], metamaterials [13], etc. If one use these materials one can effectively control emission, propagation and 
detection of the electromagnetic waves, and develop new designs of the photonic devices and those one for other parts of the electromagnetic spectrum. For instance, very active research worldwide is concentrated currently on the $\mathrm{THz}$ range, attempting to overcome the so called problem of the terahertz gap [14].

The analysis of propagation of the electromagnetic waves in periodic $[3,11,13,15]$, quasiperiodic $[12,16,17]$ and random [18] layered media is a problem which extends over the all fields in the modern physics. Optics is the area where it is crucial to calculate spectral characteristics, absorbance coefficients, polarization properties and other features of the multilayer structures in a wide spectral range and at various thicknesses or material properties of constituents. Even in the fiber optics, where usually propagation characteristics of the optical pulses $[19,20]$ are considered, the spectral characteristics become of the principal interest when optical channel incorporates such inhomogeneities as fiber Bragg gratings and fiber knots, and microresonators based on them.

In addition the use of the optical control techniques for phased-array antennas [21-22] promises to alleviate many of the problems associated with traditional electronic steering systems. The unique properties of layered anisotropic photonic structures (for example see [11]) are suitable for this application.

Here we discuss the optical properties of one-dimensional arbitrarily anisotropic photonic multilayers. The main objective of the chapter is the obtaining of a solution to the numerical problem of the electromagnetic plane wave interaction with arbitrarily anisotropic and arbitrarily inhomogeneous one-dimensional photonic structures. It is well known that many of novel technological designs have resulted from analysis of the properties of materials and creation of new structural configurations for them. In order to develop a new structural configuration with unique properties, one needs to thoroughly understand the characteristics of the structure. This can be accomplished by applying an advanced computational engine.

Today several numerical techniques are commonly used to compute the spectral characteristics of the layered photonic structures and electromagnetic field distribution in the interiors [3,12-15,23-31]. The most known and, probably, most usable are the finite element method (FEM) $[3,24]$, the transfer matrix method (TMM) [25,26], the finite difference time-domain method (FDTD) $[15,27]$ and the beam propagation method (BPM) [28]. Unfortunately, some methods are entirely disregarding the anisotropy and the inhomogeneity of the constituent materials. The most of methods which do account for material anisotropy require that the permittivity tensor be diagonal. Others allow for nondiagonal tensors, but require that the offdiagonal elements be small in comparison to the diagonal terms [28]. Although these techniques are adequate for many layered structures, they cannot be easily applied to multilayers in which the anisotropy is arbitrary oriented along an oblique axis on the random layers. In contrast to these numerical methods the method discussed here makes it possible the analyzing of inhomogeneousone-dimensional anisotropic multilayers with an arbitrary permittivity tensor and the optical axis arbitrarily oriented on any layer of LPS.

A general theory of electromagnetic propagation in periodic anisotropic layered media has been treated by a number of authors [26, 29-31]. The present chapter describes the efficient 
physico-mathematical model pertinent to one-dimensional optical-range microstructures based upon anisotropic materials. The electromagnetic field scalarization procedure [29-30] is used after the initial vector electromagnetic diffraction problem is reduced to the boundary problem for two scalar potentials. As a result, a set of linear algebraic equations are obtained.By solving them we find the unknown transmission and reflection spectra for the structure under study. The major advantage of the proposed method is that homogeneous, piecewise homogeneous and continuously inhomogeneous flat-layered anisotropic media can be analyzed on the same footing.

The chapter is organized as follows: in Section 2 we present the method of calculation employed in the chapter, which is based on electromagnetic field scalarization procedure in conjunction with the finite-difference method; Section 3 is devoted to the presentation of the numerical results, together with the discussion of their main features; we summarize our study and conclude the chapter in Section 4.

\section{Theoretical model}

In this section we present the mathematical background for calculation spectral characteristics of anisotropic layered media. The presented theory is applicable to any anisotropic layers with arbitrary orientation of the optical axes on each layer and for arbitrary angle of incidence.

The structure under consideration is schematically depicted in Fig. 2. Let's introduce the Cartesian coordinate system $x, y, z$ such that the $z$ axis is directed vertically upward. In this coordinate system, an inhomogeneous anisotropic layered medium is represented by the single layer that occupies the domain $-b<z<0,-\infty<x, y<+\infty$. The upper free half-space $z>0$ and substrate $z<-b$ are homogeneous and isotropic and have permittivities $\varepsilon_{0}, \mu_{0}$ and $\varepsilon_{c^{\prime}} \mu_{c^{\prime}}$ respectively. In general case, all layers in this geometry are lossy media.

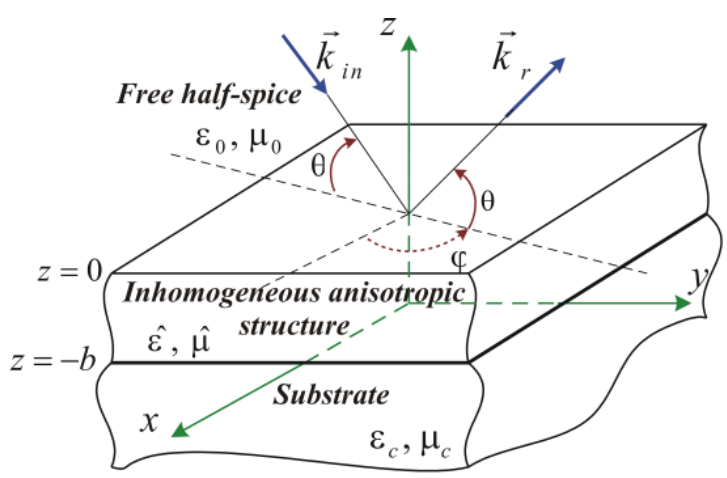

Figure 2. The benchmark photonic structure

The layered character of the media consists in next conditions or combination of them: 
- permeability $\hat{\mu}$ and permittivity $\hat{\varepsilon}$ are continuous functions of the variable $z$. These complexvalued tensors $\hat{\eta}=\hat{\varepsilon}, \hat{\mu}$ can be expressed in Cartesian coordinates as:

$$
\hat{\eta}=\left[\begin{array}{ccc}
\eta_{x x} & \eta_{x y} & \eta_{x y} \\
\eta_{y x} & \eta_{y y} & \eta_{y z} \\
\eta_{z x} & \eta_{z y} & \eta_{z z}
\end{array}\right]
$$

- the media are piecewise continuous, i.e. there are boundary surfaces $z=z_{j}=$ const, $\quad($ const $>0)$, where properties of anisotropic media are varying stepwise;

- in the points $z=0$ and $z=-b,(b>0)$ the medium is bounded by homogeneous conducting planes or the planes permeable for the electromagnetic field. In our case the layered medium is confined by the impedance planes. These planes are characterized by impedance dyads $\hat{L}^{(a)}$ and $\hat{L}^{(u)}$ :

$$
\hat{L}^{(a, u)}=\left[\begin{array}{ll}
\hat{L}_{x x}^{(a, u)} & \hat{L}_{x y}^{(a, u)} \\
\hat{L}_{y x}^{(a, u)} & \hat{L}_{y y}^{(a, u)}
\end{array}\right] .
$$

Here indices $a$ (above) and $u$ (under) correspond to upper $(z=0)$ and lower $(z=-b)$ boundaries, respectively.

We will start with Maxwell's equations, for complex field vectors $\vec{E}(\vec{R})$ and $\vec{H}(\vec{R})$, in the form:

$$
\begin{aligned}
& \nabla \times \vec{E}(\vec{R})-i k_{0} \hat{\mu}(z) \vec{H}(\vec{R})=-(4 \pi / c) \vec{M}(\vec{R}) \\
& \nabla \times \vec{H}(\vec{R})+i k_{0} \hat{\varepsilon}(z) \vec{E}(\vec{R})=(4 \pi / c) \vec{J}(\vec{R})
\end{aligned}
$$

where $k_{0}$ is the wave number in free space; $c$ is the velocity of light; $\vec{J}(\vec{R})$ and $\vec{M}(\vec{R})$ are electric and magnetic volume current densities, respectively; $\vec{R}=(x, y, z)$ is the radius vector. Here we assume the harmonic time dependence $\exp (-i \omega t)$ of the fields. Equations (3) are satisfied everywhere within the medium except the interfaces.

The electric and magnetic fields must satisfy the suitable boundary condition at the interfaces of the layered media:

- at the first, the tangential components of the electromagnetic field must be continuous at the all boundaries of the layered media:

$$
\left\{z_{0} \times \vec{E}_{\perp}\right\}=0,\left\{z_{0} \times \vec{H}_{\perp}\right\}=0,\left(z=z_{j}, j=1,2, \ldots, N\right)
$$


where $\vec{z}_{0}$ is unit vector along $z$ axis; $\vec{E}_{\perp} \equiv \vec{E}_{\perp}(\vec{R}), \vec{H}_{\perp} \equiv \vec{H}_{\perp}(\vec{R})$ are electric and magnetic field components that are orthogonal to $\vec{z}_{0}$. Throughout the chapter we use braces \{\} for next operator designation $\{f(z)\} \equiv f(z+0)-f(z-0)$;

- at the second, we introduce impedance boundary conditions that are desired on the above and the bottom boundaries of the inhomogeneous anisotropic structure:

$$
\begin{aligned}
& \vec{E}_{\perp}+\hat{L}^{(a)} \vec{z}_{0} \times \vec{H}_{\perp}=0,(z=0) ; \\
& \vec{E}_{\perp}-\hat{L}^{(u)} \vec{z}_{0} \times \vec{H}_{\perp}=0,(z=-b) .
\end{aligned}
$$

The next one, in what follows we assumed that external sources and electromagnetic field components are represented by spatial harmonics with wave vector $\vec{\kappa}=\left(\kappa_{x}, \kappa_{y}, 0\right)$

$$
\begin{aligned}
& \vec{J}(\vec{R}) \equiv \vec{J}(\vec{\kappa}, z) \exp (i \vec{\kappa} \cdot r) \\
& \vec{M}(\vec{R}) \equiv \vec{M}(\vec{\kappa}, z) \exp (i \vec{\kappa} \cdot r) \\
& \vec{E}(\vec{R}) \equiv \vec{E}(\vec{\kappa}, z) \exp (i \vec{\kappa} \cdot r), \\
& \vec{H}(\vec{R}) \equiv \vec{H}(\vec{\kappa}, z) \exp (i \vec{\kappa} \cdot r) .
\end{aligned}
$$

In expressions (6) - (7) $\kappa_{x, y}$ are the arbitrary complex constants, $\vec{J}(\vec{\kappa}, z), \vec{M}(\vec{\kappa}, z)$ and $\vec{E}(\vec{\kappa}, z)$, $\vec{H}(\vec{\kappa}, z)$ are the vector amplitudes of the sources and the fields, respectively.

Now let's try to obtain general expressions for the transmittance and reflectance of a layered medium.

\subsection{Reduction of the electromagnetic field diffraction problem to a boundary value problem for scalar potentials}

Now let's consider in details the solving of the initial electromagnetic field diffraction problem. At first we should to introduce right-hand basis of vectors $\vec{a}_{z}, \vec{a}_{l}, \vec{a}_{t}$ :

$$
\begin{aligned}
& \vec{a}_{z}=\vec{z}_{0}, \\
& \vec{a}_{l}=\vec{n}, \\
& \vec{a}_{t}=\vec{z}_{0} \times \vec{n} .
\end{aligned}
$$

In writing eq (8) we have assumed that: $\vec{n}=\vec{\kappa} / \kappa$ is the unit vector, that is situated in the plane $z=0 ; \kappa=\sqrt{\kappa_{x}^{2}+\kappa_{y}^{2}}$ is the branch of the square root, which is chosen such that condition $0 \leq \arg \sqrt{(\cdot)} \leq \pi$ shall be satisfied. These unit vectors obey the orthogonality relations: 


$$
\vec{a}_{\sigma} \cdot \vec{a}_{\tau}=0, \quad(\sigma \neq \tau)
$$

Then after scalar multiplication of the Maxwell's equations (3) by unit vectors $\vec{a}_{z}, \vec{a}_{l}$ and using simple conversion of vector algebra we obtain $E_{z, l}=\vec{a}_{z, l} \cdot \vec{E}(\vec{k}, z), H_{z, l}=\vec{a}_{z, l} \cdot \vec{H}(\vec{k}, z)$ in terms of scalar potentials:

$$
\begin{aligned}
& e(\vec{\kappa}, z)=\vec{a}_{t} \cdot \vec{E}(\vec{\kappa}, z), \\
& h(\vec{\kappa}, z)=\vec{a}_{t} \cdot \vec{H}(\vec{\kappa}, z) .
\end{aligned}
$$

In the basis of orthogonal vectors (8), vector amplitudes $\vec{E}(\vec{\kappa}, z), \vec{H}(\vec{\kappa}, z)$ can be written down as:

$$
\begin{aligned}
& \vec{E}(\vec{\kappa}, z)=\vec{V}_{\varepsilon}(\vec{n}, z) e(\vec{\kappa}, z)-\vec{W}_{\varepsilon}(\vec{\kappa}) h(\vec{\kappa}, z)+\left(4 \pi i / k_{0} c\right) \hat{\alpha}_{\varepsilon}(\vec{n}, z) \cdot \vec{J}(\vec{\kappa}, z), \\
& \vec{H}(\vec{\kappa}, z)=\vec{V}_{\mu}(\vec{n}, z) h(\vec{\kappa}, z)-\vec{W}_{\mu}(\vec{\kappa}) e(\vec{\kappa}, z)+\left(4 \pi i / k_{0} c\right) \hat{\alpha}_{\mu}(\vec{n}, z) \cdot \vec{M}(\vec{\kappa}, z) .
\end{aligned}
$$

The following symbols are used in (11): $\vec{V}_{\eta}(\vec{n}, z)$ and $\hat{\alpha}_{\eta}(\vec{n}, z),(\eta=\varepsilon, \mu)$ are the vector functions and the dyad functions, respectively; $\vec{W}_{\eta}(\vec{\kappa})$ - vector differential operator:

$$
\begin{gathered}
\vec{V}_{\eta}(\vec{n}, z)=\vec{z}_{0} \times \vec{n}+1 / a_{\eta}(\vec{n}, z)\left[b_{\eta}(\vec{n}, z) \vec{n}+c_{\eta}(\vec{n}, z) \vec{z}_{0}\right], \\
a_{\varepsilon}(\vec{n}, z) \hat{a}_{\varepsilon}(\vec{n}, z) \equiv a_{t} \times \varepsilon(z) \times a_{t}=\left(\varepsilon_{l z} \vec{n}-\varepsilon_{l l} \vec{z}_{0}\right) \vec{z}_{0}+\left(\varepsilon_{z l} \vec{z}_{0}-\varepsilon_{z z} \vec{n}\right) \vec{n}, \\
\vec{W}_{\varepsilon}(\vec{\kappa})=\left(1 / a_{\eta}(\vec{n}, z) k_{0}\right) \times\left[\left(\varepsilon_{z z} \vec{n}-\varepsilon_{z l} \vec{z}_{0}\right) i \partial_{z}+\left(\varepsilon_{z l} \vec{z}_{0}-\varepsilon_{z z} \vec{n}\right) k_{0}\right], \\
a_{\varepsilon} \rightarrow a_{\mu}, \quad \hat{a}_{\varepsilon} \rightarrow \hat{a}_{\mu}, \quad \hat{W}_{\varepsilon} \rightarrow \hat{W}_{\mu}, \quad(\varepsilon \rightarrow \mu) .
\end{gathered}
$$

In expressions (12) - (15), scalars $\varepsilon_{\sigma \tau} \equiv \varepsilon_{\sigma \tau}(\vec{n}, z), \mu_{\sigma \tau} \equiv \mu_{\sigma \tau}(\vec{n}, z), \quad(\sigma, \tau=z, l, t)$ are components of the relevant dyads $\hat{\varepsilon}, \hat{\mu}(1)$ :

$$
\begin{aligned}
& \varepsilon_{\sigma \tau}(\vec{n}, z)=\vec{a}_{\sigma} \cdot \varepsilon(\mathrm{z}) \cdot \vec{a}_{\tau}, \\
& \mu_{\sigma \tau}(\vec{n}, z)=\vec{a}_{\sigma} \cdot \mu(\mathrm{z}) \cdot \vec{a}_{\tau} .
\end{aligned}
$$

The scalar functions $a_{\eta}(\vec{n}, z), \quad b_{\eta}(\vec{n}, z), \quad c_{\eta}(\vec{n}, z), \quad(\eta=\varepsilon, \mu)$ that are used in $(12)-(14)$ have the form: 


$$
\begin{aligned}
& a_{\varepsilon}=\varepsilon_{z z} \varepsilon_{l l}-\varepsilon_{z l} \varepsilon_{l z}, \\
& b_{\varepsilon}=\varepsilon_{z t} \varepsilon_{l z}-\varepsilon_{z z} \varepsilon_{l t}, \\
& c_{\varepsilon}=\varepsilon_{l t} \varepsilon_{z l}-\varepsilon_{l l} \varepsilon_{z t},
\end{aligned}
$$

$a_{\varepsilon} \rightarrow a_{\mu^{\prime}} \quad b_{\varepsilon} \rightarrow b_{\mu^{\prime}} \quad c_{\varepsilon} \rightarrow c_{\mu}, \quad(\varepsilon \rightarrow \mu)$.

On the next step after scalar multiplication of the Maxwell's equations (3) by the unit vector $\vec{a}_{t}$, and using the expressions (11) we obtain:

$$
\begin{aligned}
& D_{s S}(\vec{\kappa}) h(\vec{\kappa}, z)+D_{s p}(\vec{\kappa}) e(\vec{\kappa}, z)=(4 \pi / c) q_{s}(\vec{\kappa}, z), \\
& D_{p p}(\vec{\kappa}) e(\vec{\kappa}, z)+D_{p s}(\vec{\kappa}) h(\vec{\kappa}, z)=(4 \pi / c) q_{p}(\vec{\kappa}, z) .
\end{aligned}
$$

Expressions (18) represent the system of coupled ordinary differential equations for two scalar potentials $e(\vec{\kappa}, z)$ and $h(\vec{\kappa}, z)$ within the interval $-b<z<0$; the external sources are entered into the quantities $q_{v}(\vec{\kappa}, z) ; D_{v \xi}(\vec{\kappa})$ are the scalar operators that depend on $\vec{\kappa}(v, \xi=s, p)$. These operators in explicit form are written as follows:

$$
\begin{gathered}
q_{s}(\vec{\kappa}, z)=i k_{0}\left[-\vec{z}_{0} \times \vec{n}+\frac{1}{a_{\mu}(\vec{n}, z)}\left(e_{\mu} \vec{z}_{0}+d_{\mu} \vec{n}\right)\right] \cdot \vec{M}(\vec{\kappa}, z)+ \\
+\left[\left(\partial_{z} \varepsilon_{l z}+i \kappa \varepsilon_{l l}\right) \vec{z}_{0}-\left(\partial_{z} \varepsilon_{z z}+i \kappa \varepsilon_{z l}\right) \vec{n}\right] \cdot \frac{\vec{J}(\vec{\kappa}, z)}{a_{\varepsilon}(\vec{n}, z)}, \\
q_{s} \rightarrow q_{p}(\varepsilon \leftrightarrow \mu, \vec{J} \rightarrow \vec{M}, \quad \vec{M} \rightarrow-\vec{J}) ; \\
D_{s s}(\vec{\kappa})=\frac{\partial}{\partial z} \frac{\varepsilon_{z z}}{a_{\varepsilon}(\vec{n}, z)} \frac{\partial}{\partial z}+i \kappa\left[\frac{\partial}{\partial z} \frac{\varepsilon_{l z}}{a_{\varepsilon}(\vec{n}, z)}+\frac{\varepsilon_{z l}}{a_{\varepsilon}(\vec{n}, z)} \frac{\partial}{\partial z}\right]+k_{0}^{2} \frac{|\hat{\mu}(z)|}{a_{\mu}(\vec{n}, z)}-\kappa^{2} \frac{\varepsilon_{l l}}{a_{\varepsilon}(\vec{n}, z)} ; \\
i k_{0}^{-1} D_{s p}(\vec{\kappa})=i \kappa\left[\frac{c_{\varepsilon}(\vec{n}, z)}{a_{\varepsilon}(\vec{n}, z)}+\frac{e_{\mu}(\vec{n}, z)}{a_{\mu}(\vec{n}, z)}\right]-\frac{\partial}{\partial z} \frac{b_{\varepsilon}(\vec{n}, z)}{a_{\varepsilon}(\vec{n}, z)}-\frac{\partial}{\partial z} \frac{e_{\mu}(\vec{n}, z)}{a_{\mu}(\vec{n}, z)} \\
D_{s S} \rightarrow D_{p p}, D_{s p} \rightarrow D_{p s^{\prime}}(\varepsilon \leftrightarrow \mu)
\end{gathered}
$$

where:

$$
\begin{aligned}
& d_{\varepsilon}=\varepsilon_{z z} \varepsilon_{t l}-\varepsilon_{z l} \varepsilon_{t z^{\prime}} \\
& e_{\varepsilon}=\varepsilon_{l l} \varepsilon_{t z}-\varepsilon_{l z} \varepsilon_{t l} \\
& d_{\varepsilon} \rightarrow d_{\mu}, e_{\varepsilon} \rightarrow e_{\mu},(\varepsilon \rightarrow \mu)
\end{aligned}
$$


After the substituting expressions (11) into condition (4) we obtain boundary conditions for scalar potentials $e(\vec{\kappa}, z), h(\vec{\kappa}, z)$ that satisfied on all boundaries $z=z_{j}$ :

$$
\begin{gathered}
\{e(\vec{\kappa}, z)\}=0, \quad\{h(\vec{\kappa}, z)\}=0, \\
\left\{1 / a_{\mu}(\vec{n}, z) \times\left[\left(\mu_{z z} \partial_{z}-i \vec{\kappa} \mu_{l z}\right) e(\vec{\kappa}, z)-i k_{0} b_{\mu}(\vec{n}, z) h(\vec{\kappa}, z)\right]\right\}=0, \\
\left\{1 / a_{\varepsilon}(\vec{n}, z) \times\left[\left(\varepsilon_{z z} \partial_{z}-i \vec{\kappa} \varepsilon_{l z}\right) h(\vec{\kappa}, z)-i k_{0} b_{\varepsilon}(\vec{n}, z) e(\vec{\kappa}, z)\right]\right\}=0 .
\end{gathered}
$$

Substituting expressions (11), for vector amplitudes $\vec{E}(\vec{\kappa}, z), \vec{H}(\vec{\kappa}, z)$, into impedance boundary conditions (5) we obtain two pair of equations for scalar potentials and their derivates. The first pair of equations for lower boundary $z=-b+0$ has the form:

$$
\begin{aligned}
& {\left[i k_{0} a_{p p}^{(u)}(\vec{\kappa})+b_{p p}^{(u)}(\vec{\kappa}) \partial_{z}\right] e(\vec{\kappa}, z)+i k_{0} a_{p s}^{(u)}(\vec{\kappa}) h(\vec{\kappa}, z)=0,} \\
& {\left[i k_{0} a_{s p}^{(u)}(\vec{\kappa})+b_{s p}^{(u)}(\vec{\kappa}) \partial_{z}\right] e(\vec{\kappa}, z)+\left[i k_{0} a_{s s}^{(u)}(\vec{\kappa})+b_{s s}^{(u)}(\vec{\kappa}) \partial_{z}\right] h(\vec{\kappa}, z)=0,}
\end{aligned}
$$

where:

$$
\begin{aligned}
& a_{p p}^{(u)}(\vec{\kappa})=1+\frac{\kappa \mu_{l z}(\vec{\kappa}, z)}{k_{0} a_{\mu}(\vec{n}, z)} L_{t t}^{(u)}(\vec{\kappa}), \\
& b_{p p}^{(u)}(\vec{\kappa})=\frac{\mu_{z z}(z)}{a_{\mu}(\vec{n}, z)} L_{t t}^{(u)}(\vec{\kappa}), \\
& a_{p s}^{(u)}(\vec{\kappa})=L_{t l}^{(u)}(\vec{\kappa})+\frac{b_{\mu}(\vec{n}, z)}{a_{\mu}(\vec{n}, z)} L_{t t}^{(u)}(\vec{\kappa}), \\
& b_{s s}^{(u)}(\vec{\kappa})=\frac{\varepsilon_{z z}(z)}{a_{\varepsilon}(\vec{n}, z)}, \\
& b_{s p}^{(u)}(\vec{\kappa})=\frac{\mu_{z z}(z)}{a_{\mu}(\vec{n}, z)} L_{l t}^{(u)}(\vec{\kappa}), \\
& a_{s p}^{(u)}(\vec{\kappa})=\frac{b_{\varepsilon}(\vec{n}, z)}{a_{\varepsilon}(\vec{n}, z)}+\frac{\kappa \mu_{l z}(\vec{\kappa}, z)}{k_{0} a_{\mu}(\vec{n}, z)} L_{l t}^{(u)}(\vec{\kappa}), \\
& a_{s s}^{(u)}(\vec{\kappa})=\frac{\kappa \varepsilon_{l z}(\vec{\kappa}, z)}{k_{0} a_{\varepsilon}(\vec{n}, z)}-\frac{b_{\mu}(\vec{n}, z)}{a_{\mu}(\vec{n}, z)} L_{l t}^{(u)}(\vec{\kappa})+L_{l l}^{(u)}(\vec{\kappa}) .
\end{aligned}
$$

In expressions (26) the equality is used $L_{\sigma \tau}^{(u)}(\vec{\kappa})=\vec{a}_{\sigma} \cdot \hat{L}^{(u)} \cdot \vec{a}_{\tau}(\sigma, \tau=z, l, t)$. 
The boundary conditions for top plane $(z=-0)$ are similar to (25). They are resulting from the following replacements in $(25)-(26): b_{p p}^{(u)}(\vec{\kappa}) \rightarrow-b_{p p}^{(a)}(\vec{\kappa}), a_{p s}^{(u)}(\vec{\kappa}) \rightarrow-a_{p s}^{(a)}(\vec{\kappa}), \quad b_{s p}^{(u)}(\vec{\kappa}) \rightarrow-b_{s p}^{(a)}(\vec{\kappa})$, $b_{s s}^{(u)}(\vec{\kappa}) \rightarrow-b_{s s}^{(a)}(\overrightarrow{\mathcal{\kappa}})$; in formulas for $a_{p p}^{(u)}(\vec{\kappa}), a_{s p}^{(u)}(\vec{\kappa}), a_{s s}^{(u)}(\vec{\kappa})$ upper index $u \rightarrow a$ and $\kappa \rightarrow-\kappa$.

\subsection{Numerical solutions by finite-difference method}

Now let's build a numerical solution of the problem of monochromatic plane wave diffraction on the inhomogeneous anisotropic layered structure. We will assume that the structure is piecewise homogeneous along the axis $z$ and within each homogeneous layer the anisotropic material is gyrotropic one, or, particularly, an uniaxial material with arbitrary orientation of the optical axes.

The electromagnetic properties of the benchmark structure in a fixed point of the space are defined by permeability and permittivity dyads:

$$
\begin{aligned}
& \hat{\varepsilon}(z)=\varepsilon_{\perp}(z) \hat{I}+\left(\varepsilon_{||}(z)-\varepsilon_{\perp}(z)\right) \vec{a} \vec{a}-i f(z) \vec{a} \times \hat{I} \\
& \hat{\mu}(z)=\mu_{\perp}(z) \hat{I}+\left(\mu_{||}(z)-\mu_{\perp}(z)\right) \vec{b} \vec{b}-i g(z) \vec{b} \times \hat{I}
\end{aligned}
$$

Here, $\varepsilon_{\perp}(z), \varepsilon_{||}(z), f(z)$ and $\mu_{\perp}(z), \mu_{\mid}(z), g(z)$ are twice differentiable functions of the variable $z ; I$ is the identity dyad; $\vec{a}$ and $\vec{b}$ are the unit vectors in the direction of the optical axes which have the following components in the Cartesian coordinate system:

$$
\begin{aligned}
& \vec{a} \equiv \vec{a}(z)=\left(\cos \theta_{a} \sin \varphi_{a}, \cos \theta_{a} \cos \varphi_{a}, \sin \theta_{a}\right), \\
& \vec{b} \equiv \vec{b}(z)=\left(\cos \theta_{b} \sin \varphi_{b}, \cos \theta_{b} \cos \varphi_{b}, \sin \theta_{b}\right) .
\end{aligned}
$$

For the sake of clarity, the sloping angles $\theta_{a^{\prime}} \theta_{b}$ and the azimuthal angles $\varphi_{a^{\prime}} \varphi_{b^{\prime}}$, which determine the optical axes direction, are shown in Fig. 3 and therewith we have:

$$
\begin{gathered}
-\pi / 2 \leq \theta_{a}, \theta_{b} \leq \pi / 2, \\
0 \leq \varphi_{a}, \varphi_{b} \leq 2 \pi .
\end{gathered}
$$

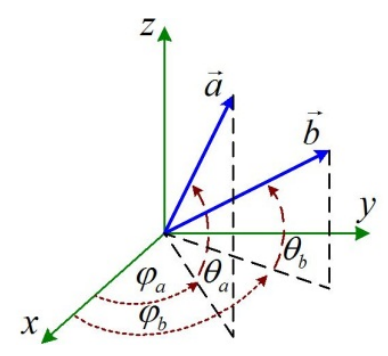

Figure 3. The optical axis orientation in the arbitrary layer 
At the same time we consider that an incident ( $s$ - or $p$-polarized) plane wave arrives from the free half-space $(z>0)$ in the direction of the unit vector $i_{i n}$ which is determined by the sloping angle $\theta$ and the azimuthal angle $\varphi$, as depicted in Fig.2. Its components in the Cartesian coordinate system are as follows:

$$
\begin{gathered}
\vec{l}_{\text {in }}=(\cos \theta \cos \varphi, \cos \theta \sin \varphi,-\sin \theta), \\
-\pi / 2 \leq \theta \leq \pi / 2, \\
0 \leq \varphi \leq 2 \pi .
\end{gathered}
$$

Let us assume the inhomogeneous anisotropic structures under consideration with thickness $b$ are placed on isotropic (or anisotropic) homogeneous substrate with permittivity and permeability $\varepsilon_{c}, \mu_{c}$. In general case, $\varepsilon_{c}$ and $\mu_{c}$ are complex values. We assume also that anisotropic layer is bounded above $(z>0)$ by free half-spice with $\varepsilon_{0}$ and $\mu_{0}$. In the case presented here, the anisotropic layer is inhomogeneous in the thickness; it means, that structure's parameters are depended on the coordinate $z$, but they are invariable along the axes $x$ and $y$. Generally these parameters are piecewise continuous functions of $z$.

Then scalar potentials $e(\vec{\kappa}, z), h(\vec{\kappa}, z)$ beyond the anisotropic layer will be presented by the following expressions:

- in the free half-spice $(0<z<+\infty)$ :

$$
\begin{aligned}
& e(\vec{\kappa}, z)=\left(e^{-i \gamma_{0} z}+R_{p p} e^{i \gamma_{0} z}\right) A_{p}+R_{p s} A_{s} e^{i \gamma_{0} z}, \\
& h(\vec{\kappa}, z)=\left(e^{-i \gamma_{0} z}+R_{s s} e^{i \gamma_{0} z}\right) A_{s}+R_{s p} A_{p} e^{i \gamma_{0} z}
\end{aligned}
$$

- in the substrate $(-\infty<z<-b)$ :

$$
\begin{aligned}
& e(\vec{\kappa}, z)=\left[T_{p s} A_{s}+T_{p p} A_{p}\right] e^{-i k_{0} n_{c}(z+b)}, \\
& h(\vec{\kappa}, z)=\left[T_{s s} A_{s}+T_{s p} A_{p}\right] e^{-i k_{0} n_{c}(z+b)} .
\end{aligned}
$$

Here $\gamma_{0}=k_{0} \sin \theta ; n_{c}=\sqrt{\varepsilon_{c} \mu_{c}-\cos \theta}, \quad\left(0 \leq \arg n_{c} \leq \pi\right)$; complex values $A_{p}$ and $A_{s}$ characterize electromagnetic (s or p-polarized) wave's components. The $s-(p-)$ polarized light corresponds to an electric (magnetic) field be parallel to the layers.

In the expressions (32) - (33) we have introduced the complex reflection $R_{v \xi}(v, \xi=p, s)$ and transmission $T_{v \xi}$ coefficients, which depend on: wave number in the free space $k_{0}$; angles $\theta, \varphi$ and other geometrical and electrodynamical parameters of the problem. The coefficients with similar lower indices $(v=\xi)$ describe conversion of the incident wave into the wave with the 
same polarization. By analogy, reflection and transmission coefficients with dissimilar lower indexes $(v \neq \xi)$ describe conversion of the incident wave into the wave with the orthogonal polarization. In that notation, the left lower index $v$ corresponds to the polarization of the reflected/transmitted wave; the right lower index $\xi$ corresponds to the polarization of the incident wave.

Notice, that the presence of the "crossed" reflection $\left(R_{s p}, R_{p s}\right)$ and transmission coefficients $\left(T_{s p}, T_{p s}\right)$, which are responsible for incident plane wave depolarization, is the specific properties of anisotropic media (see, for example [31]).

As it follows from the expressions (32) - (33):

- for the case of $s$ - polarized incident plane wave $\left(A_{s}=1, A_{p}=0\right)$ :

$$
\begin{aligned}
& R_{s S}=h(\vec{\kappa}, 0)-1, \\
& R_{p s}=e(\vec{\kappa}, 0), \\
& T_{s s}=h(\vec{\kappa},-b), \\
& T_{p s}=e(\vec{\kappa},-b) ;
\end{aligned}
$$

- for the case of $p$ - polarized incident plane wave $\left(A_{s}=0, A_{p}=1\right)$ :

$$
\begin{aligned}
& R_{p p}=e(\vec{\kappa}, 0)-1, \\
& R_{s p}=h(\vec{\kappa}, 0), \\
& T_{p p}=e(\vec{\kappa},-b), \\
& T_{s p}=h(\vec{\kappa},-b) .
\end{aligned}
$$

The impedance boundary conditions (25) for the scalar potentials can be rewritten in the following form:

$$
\begin{aligned}
& \partial h / \partial z=i k_{0}\left(\lambda_{s p}^{(a)} e+\lambda_{s s}^{(a)} h\right)+f_{s^{\prime}}, \quad(z=-0) ; \\
& \partial e / \partial z=i k_{0}\left(\lambda_{p p}^{(a)} e+\lambda_{p s}^{(a)} h\right)+f_{p^{\prime}} \\
& \partial h / \partial z=i k_{0}\left(\lambda_{s p}^{(u)} e+\lambda_{s s}^{(u)} h\right), \\
& \partial e / \partial z=i k_{0}\left(\lambda_{p p}^{(u)} e+\lambda_{p s}^{(u)} h\right),
\end{aligned}
$$


The values $\lambda_{v \xi}^{(a, u)}, f_{v}$ are depending on the angles $\theta$ and $\varphi$ and have the form:

$$
\begin{aligned}
& \lambda_{p p}^{(a)}=\frac{1}{\mu_{z z}(0)}\left[a_{\mu}(0) \sin \theta-\mu_{l z}(0) \cos \theta\right], \\
& \lambda_{p s}^{(a)}=\frac{b_{\mu}(0)}{\mu_{z z}(0)}, \\
& \lambda_{s p}^{(a)}=-\frac{b_{\varepsilon}(0)}{\varepsilon_{z z}(0)}, \\
& \lambda_{s s}^{(a)}=\frac{1}{\varepsilon_{z z}(0)}\left[a_{\varepsilon}(0) \sin \theta-\varepsilon_{l z}(0) \cos \theta\right] ; \\
& \lambda_{p p}^{(u)}=-\frac{1}{\mu_{z z}(-b)}\left[a_{\mu}(-b) \frac{n_{c}}{\mu_{c}}+\mu_{l z}(-b) \cos \theta\right], \\
& \lambda_{p s}^{(u)}=\frac{b_{\mu}(-b)}{\mu_{z z}(-b)}, \\
& \lambda_{s p}^{(u)}=-\frac{b_{\varepsilon}(-b)}{\varepsilon_{z z}(-b)}, \\
& \lambda_{s s}^{(u)}=\frac{1}{\varepsilon_{z z}(-b)}\left[a_{\varepsilon}(-b) \frac{n_{c}}{\mu_{c}}+\varepsilon_{l z}(-b) \cos \theta\right] ; \\
& f_{s}=-2 i k_{0} a_{\varepsilon}(0) \sin \theta A_{s} / \varepsilon_{z z}(0), \\
& f_{p}=-2 i k_{0} a_{\mu}(0) \sin \theta A_{p} / \mu_{z z}(0) .
\end{aligned}
$$

Let us build the finite-difference procedure that approximately describes the system of coupled differential equations (18) and the boundary conditions (36) - (37). Taking into account the fact, that the external sources are absent inside the anisotropic layer, the substitutions $q_{p}=q_{s} \equiv 0$ are required in formulas (18).

At first, we divide the segment $-b<z<0$ on the $N$ equal parts. After that the grid step is immediately obtained as $\Delta b=b / N$, and then the grid's knot set $z_{0}, z_{1}, \ldots, z_{N}$ is defined by formula $z_{j}=j \Delta b-b, \quad(j=0,1, \ldots, N)$. Notice, that this knot set includes boundary points $z_{0}=-b$ and $z_{N}=0$ as well. The partial derivatives in the differential equations (18) are approximated by the central difference; in the boundary conditions (36) - (37), they are approximated by the left-hand difference and the right-hand difference, correspondingly. Then, we obtain the system of linear algebraic equations with dimension $2 N+2$ for the unknown complex variables $x_{k} \equiv h\left(\vec{\kappa}, z_{k}\right), y_{k} \equiv e\left(\vec{\kappa}, z_{k}\right), \quad(k=0,1, \ldots, N)$. For the case of $s$-polarized wave this system has the form: 


$$
\begin{gathered}
\left\{\begin{array}{l}
x_{N}\left(1-i k_{0} b \lambda_{s s}^{(a)}\right)-x_{N-1}-i k_{0} b \lambda_{s p}^{(a)} y_{N}=f_{s s^{\prime}} \\
-i k_{0} b \lambda_{p s}^{(a)} x_{N}+y_{N}\left(1-i k_{0} b \lambda_{p p}^{(a)}\right)-y_{N-1}=0 ;
\end{array}\right. \\
\left\{\begin{array}{l}
A_{j} x_{j+1}+B_{j} x_{j}+C_{j} x_{j-1}+D_{j} y_{j+1}+F_{j} y_{j}+G_{j} y_{j-1}=0, \\
P_{j} x_{j+1}+Q_{j} x_{j}+R_{j} x_{j-1}-K_{j} y_{j+1}-L_{j} y_{j}-M_{j} y_{j-1}=0,
\end{array}\right. \\
(j=1,2, \ldots, N-1) ; \\
\left\{\begin{array}{l}
x_{1}-x_{0}\left(1+i k_{0} b \lambda_{s s}^{(u)}\right)-i k_{0} b \lambda_{s p}^{(u)} y_{0}=f_{s s^{\prime}} \\
y_{1}-y_{0}\left(1+i k_{0} b \lambda_{p p}^{(u)}\right)-i k_{0} b \lambda_{p s}^{(u)} y_{0}=0 .
\end{array}\right.
\end{gathered}
$$

Here, the first equation set (41) is corresponding to the finite-difference approximation of the boundary conditions (36) imposed at $z=0, A_{s}=1, A_{p}=0$. The next equation set (42) is the finitedifference approximation of the differential equations (18) and the next one equation set (43) is the finite-difference approximation of the boundary conditions (37) imposed $z=-b ; f_{s s}=\Delta b f_{s}$.

The coefficients $A_{j}, \ldots, M_{j}$ that enter the equations (42) are given by the expressions:

$$
\begin{gathered}
A_{j}=\frac{\varepsilon_{z z}\left(z_{j}\right)}{a_{\varepsilon}\left(z_{j}\right)}+\frac{1}{4}\left[\frac{\varepsilon_{z z}\left(z_{j+1}\right)}{a_{\varepsilon}\left(z_{j+1}\right)}-\frac{\varepsilon_{z z}\left(z_{j-1}\right)}{a_{\varepsilon}\left(z_{j-1}\right)}\right]+\frac{i k_{0} b \cos \theta}{2}\left[\frac{\varepsilon_{z l}\left(z_{j}\right)}{a_{\varepsilon}\left(z_{j}\right)}+\frac{\varepsilon_{l z}\left(z_{j+1}\right)}{a_{\varepsilon}\left(z_{j+1}\right)}\right], \\
B_{j}=-2 \frac{\varepsilon_{z z}\left(z_{j}\right)}{a_{\varepsilon}\left(z_{j}\right)}+\left(k_{0} b\right)^{2}\left[\frac{\left|\mu\left(z_{j}\right)\right|}{a_{\mu}\left(z_{j}\right)}-\cos ^{2} \theta \frac{\varepsilon_{l l}\left(z_{j}\right)}{a_{\mu}\left(z_{j}\right)}\right], \\
D_{j}=\frac{i k_{0} b}{2}\left[\frac{d_{\mu}\left(z_{j}\right)}{a_{\mu}\left(z_{j}\right)}+\frac{b_{\varepsilon}\left(z_{j+1}\right)}{a_{\varepsilon}\left(z_{j+1}\right)}\right], \\
C_{j}=\frac{\varepsilon_{z z}\left(z_{j}\right)}{a_{\varepsilon}\left(z_{j}\right)}-\frac{1}{4}\left[\frac{\varepsilon_{z z}\left(z_{j+1}\right)}{a_{\varepsilon}\left(z_{j+1}\right)}-\frac{\varepsilon_{z z}\left(z_{j-1}\right)}{a_{\varepsilon}\left(z_{j-1}\right)}\right]-\frac{i k_{0} b \cos \theta}{2}\left[\frac{\varepsilon_{z l}\left(z_{j}\right)}{a_{\varepsilon}\left(z_{j}\right)}-\frac{\varepsilon_{l z}\left(z_{j-1}\right)}{a_{\varepsilon}\left(z_{j-1}\right)}\right], \\
F_{j}=-\left(k_{0} b\right)^{2} \cos \theta\left[\frac{c_{\varepsilon}\left(z_{j}\right)}{a_{\mu}\left(z_{j}\right)}-\frac{e_{\mu}\left(z_{j}\right)}{a_{\mu}\left(z_{j}\right)}\right], \\
G_{j}=-\frac{i k_{0} b}{2}\left[\frac{d_{\varepsilon}\left(z_{j}\right)}{a_{\mu}\left(z_{j}\right)}-\frac{b_{\varepsilon}\left(z_{j-1}\right)}{a_{\varepsilon}\left(z_{j-1}\right)}\right], \\
A_{j} \rightarrow K_{j}, \quad B_{j} \rightarrow L_{j}, \quad C_{j} \rightarrow M_{j}, \quad D_{j} \rightarrow P_{j}, \quad F_{j} \rightarrow Q_{j}, \quad G_{j} \rightarrow R_{j}, \quad(\varepsilon \leftrightarrow \mu) .
\end{gathered}
$$

The system of equations for the $p$-polarized incident plane wave, can be received from (41) (43) by the substitutions $f_{s s} \rightarrow 0$ and $0 \rightarrow f_{p p}=\Delta b f_{p}$ in the systems (41), (43). For the case, when anisotropic layer is uniaxial media: $f(z)=g(z) \equiv 0$. 
As a result, a set of linear algebraic equations (41) - (43) with dimension $2 N+2$ is derived. Obtained linear system of equations can be solved by standard techniques such as Gauss method. By solving it we find the unknown transmission and reflection factors for the structure under study.

\section{Results and discussion}

In this section we present the results of numerical simulations that illustrate the influence of anisotropy and inhomogeneities of the materials composed of layers in the structure being studied upon the mechanisms of diffraction of the incident plane electromagnetic wave.

All structures presented here are based on the porous silicon (PSi). Today, PSi plays an important role in a number of applications. These include microcavities [11,32], photonic crystals [33], waveguide structures [34], photodetectors [35], sensors [36], etc. Besides, PSi has the potential to be an optically active material in the case when an acceptable electro- or thermooptic media is infiltrated into the pores [32]. Therefore, porous silicon is an excellent candidate for tunable optical interconnects and optical switches. For all these applications a strict control over the reflectance and transmission properties of PSi layers is required.

Today porous silicon attracts a great deal of attention because it's a material with great technological promise. The main advantages of PSi may be summarized as follows:

- PSi is a simple and low cost dielectric material that can be easy prepared;

- PSi is a promising material for photonic applications due to its excellent thermal and mechanical properties, obvious compatibility with standard Si-based technologies;

- PSi is a suitable material for the formation of arbitrary multilayers. PSi multilayers are almost arbitrary combination of layers with different thickness and porosity (refractive index), because these two parameters can be relatively easily controlled during the formation process of porous silicon.

It is well known that the PSi films can be produced by anode electrochemical etching of the monocrystalline silicon plates [37]. The nanometer-size pores tend to grow in the direction of electrochemical etching and, accordingly, nanocrystal formation sets in. The porosity $P$ and the effective refractive index $n_{\text {eff }}=\sqrt{\varepsilon_{\text {eff }}}$ of PSi are controlled by the current density under electrochemical etching, because the effective refractive index of PSi is determined by the porosity and refractive index of the medium inside the pores. Thus, by periodically varying the magnitude of current density we are able to obtain the structure with alternating layers of different porosity and, consequently, with different refractive indices.

The bulk silicon crystal is not birefringent due to its cubic crystal symmetry. However, porous modification of silicon can exhibit strong in-plane anisotropy of the refractive index $[37,38]$. The observed birefringence depends on the porosity, the size of Si nanocrystals, the spacing between them and the dielectric properties of surrounding medium. As was demonstrated in 
[39], PSi layers, with dimension of the pore about 10-30 nm, have properties of the negative uniaxial crystal with diagonal permittivity tensor

$$
\varepsilon=\left[\begin{array}{ccc}
n_{0}^{2} & 0 & 0 \\
0 & n_{0}^{2} & 0 \\
0 & 0 & n_{e}^{2}
\end{array}\right],
$$

whose birefringence magnitude $\Delta n=n_{o}-n_{e}$ is up to 0.24 . In expression (45) $n_{0}$ is the index of refraction for the waves polarized perpendicularly to the optical axis, which are called "ordinary" or "o-waves"; $n_{e}$ is the index of refraction for the waves polarized parallel to the optical axis, which are called "extraordinary" or "e - waves". It is important to note that if $n_{e}>n_{0}$ the crystal is said to be positively uniaxial, in opposite case if $n_{e}<n_{0}$ the crystal is said to be negatively uniaxial. When a linearly polarized wave of arbitrary polarization direction enters an anisotropic medium, it will be split into two components polarized along the two allowed polarization directions which are determined by the direction of the wave vector relative to the axes of the indicatrix. As a result, each $s$ - or $p$ - polarized plane wave incident on such an anisotropic photonic structure will generate two reflected and two transmitted plane waves containing both $s$ - and $p$ - polarized planewaves. For the special cases when the principal axes of the layers are parallel or perpendicular to the fixed axes, the $s$-and $p$-polarized waves remain uncoupled [26].

Two types of PBG structures are investigated, namely, a distributed Bragg reflector (DBR) in other words photonic crystal (PhC) and a microcavity.

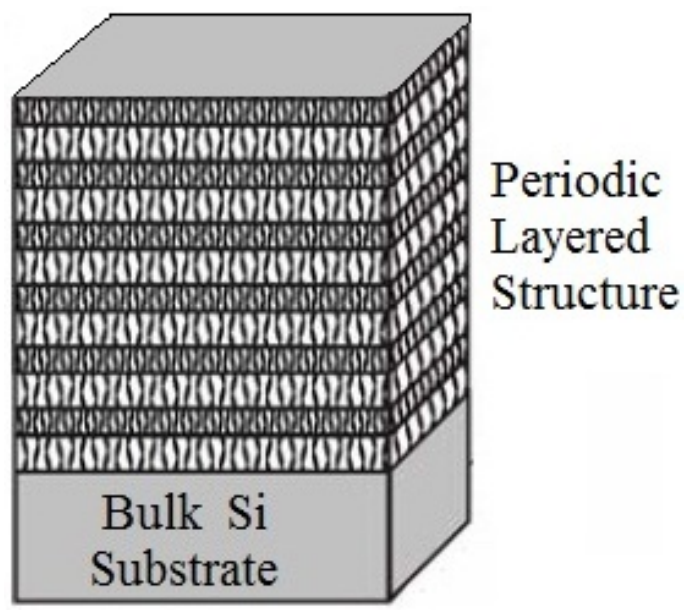

Figure 4. Schematic presentation of PSi-based PhC: the dark layers have high porosity (low refractive index) and the bright layers are of low porosity (high refractive index) 


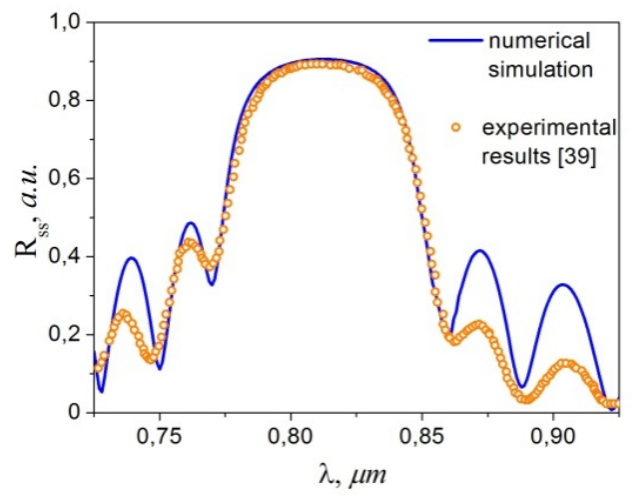

Figure 5. Reflectance spectra $R_{s s}$ of the PhC: solid line - numerical simulation; scatter - experimental results [39]. The calculations involved the value of sloping and azimutal angles: $\theta=70^{\circ}, \theta_{a}=45^{\circ}$ and $\varphi=\varphi_{a}=0^{\circ}$

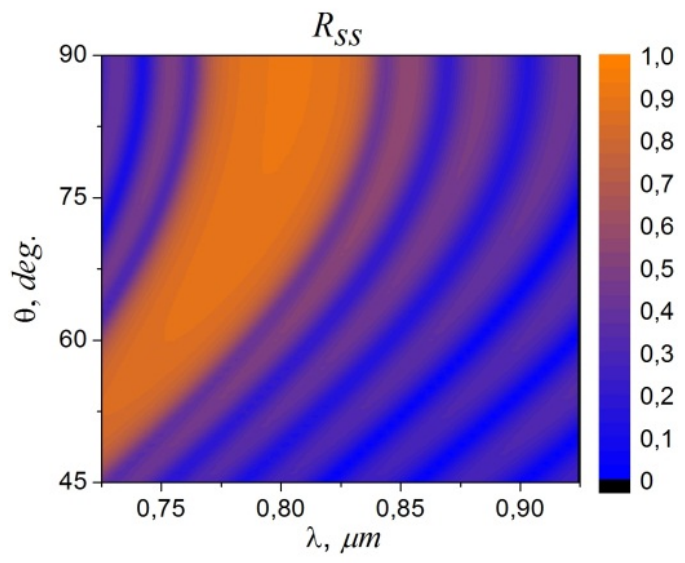

Figure 6. Reflectance spectra $R_{s s}$ as function of incident sloping angle $\theta$. In this case $\theta_{a}=45^{\circ}, \lambda_{c}=800 \mathrm{~nm}$

The simplest multilayered PBG structure is one-dimensional photonic crystal as depicted on Fig. 4. It's well known that PhCs are class of optical media represented by the natural or artificial structures with periodic modulation of the refractive index. Such optical media have some peculiar properties which gives an oportunity for a number of applications to be implemented on their basis. The most important property which determines practical significance of the PhC is the presence of the omnidirectional photonic band gap. The PBG refers to the energy or frequency range where the light propagation is prohibited inside the $\mathrm{PhC}$. As an example of such a PhC one can give a Bragg grating which is widely used as a distributed 
reflector in vertical cavity surface emitting lasers. Besides, such structures are widely used as antireflecting coatings which allow dramatically decrease the reflectance from the surface and are used to improve the quality of lenses, prisms and other optical components.

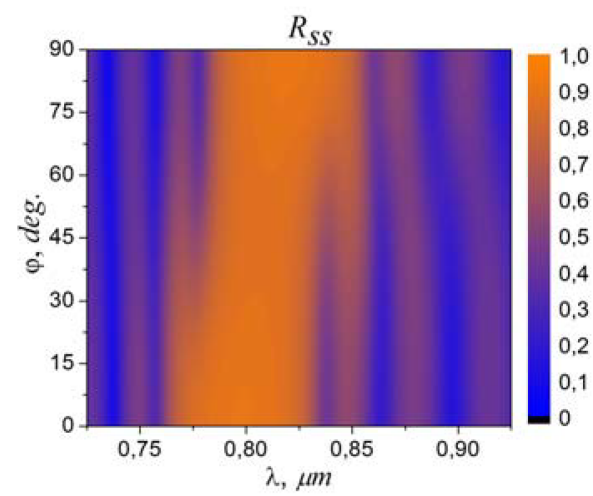

a

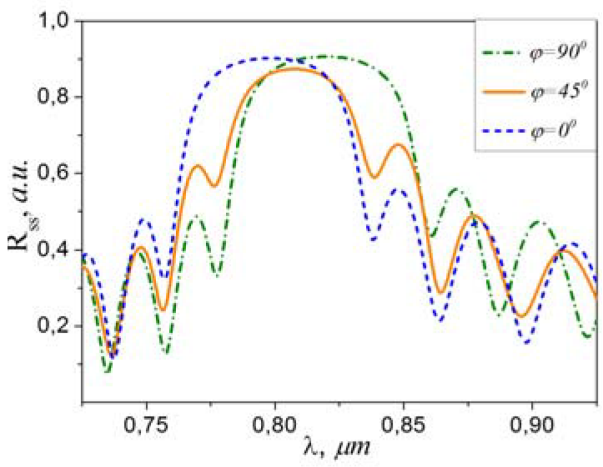

b

Figure 7. Reflectance spectra $R_{s S}$ as function of the incident azimuthal angle $\varphi$

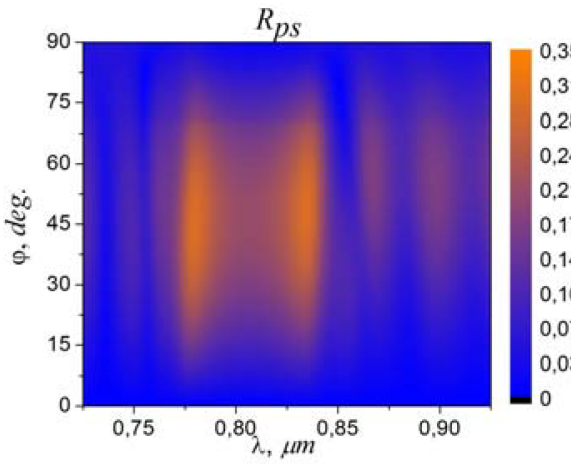

(a)

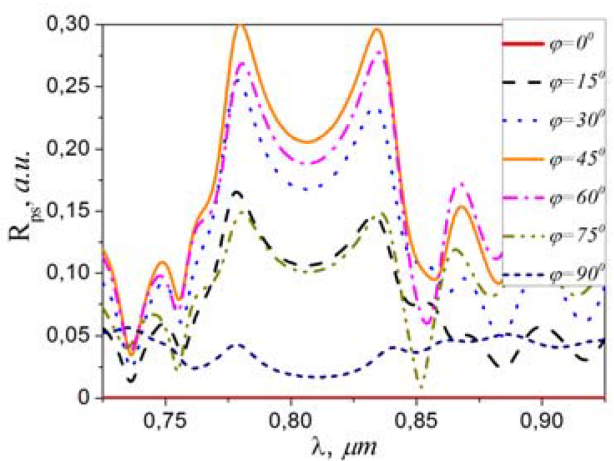

(b)

Figure 8. Reflectance spectra $R_{p s}$ as function of the incident azimuthal angle $\varphi$

First, we study light propagation in a one-dimensional photonic crystal, which was identical to $\mathrm{PhC}$ that was experimental investigated by Aktsipetrov et al. [39]. It consists of 25 pairs (building blocks) of lossy anisotropic layers with refractive indices: $n_{01}=1.39+0.008 i$, $n_{e 1}=1.32+0.008 i, n_{o 2}=1.58+0.008 i, n_{e 2}=1.5+0.008 i$. The materials are assumed to be nonmagnetic so that $\mu=1$ throughout the whole layered medium. The physical thickness of the layers 
was chosen such that the optical thickness of layers was equal to $\lambda_{c} / 4$, where $\lambda_{c}=800 \mathrm{~nm}$ is the Bragg wavelength corresponding to the photonic band gap (PBG) centre at the normal incidence $(\theta=90)$. The numerical modeling were made for the case where the optical axes of all structure layers were oriented in one and the same direction.

The comparison of the results of the proposed numerical scheme with results of the cited experimental work [39] is presented on Fig. 5. The reflectance spectra demonstrate existence of a PBG with a reflectance of about 0.9 in the wavelength region of 770-860 nm. As we see theoretical result is in very good agreement with the experimental one. So proposed method can be successfully used for computation of the spectral characteristics of $1 \mathrm{D}$ anisotropic layered structures.

For all the structures discussed here, the reflection/transmission spectrum is strongly dependent on the incident sloping $\theta$ and azimuthal $\varphi$ angles. The dependences of the reflection spectra of PSi-based distributed Bragg reflectors from variation of the sloping angle $\theta$ and the azimuthal angle $\varphi$ of the incident $s$-polarized plane wave are shown in Fig. 6. - Fig. 8. In all cases we take $\lambda_{c}=800 \mathrm{~nm}$.

It may be seen from the Fig. 6 that in agreement with the theory the width of the high reflectance region (width of the photonic band gap) is decreased with decreasing the sloping angle $\theta$, and the central wavelength of PBG is shifted to the short wavelength region. In this case we assume that $\varphi=\varphi_{a}=0^{0}$ in result the conversion incident wave to wave with orthogonal polarization is absent.

The influence of the azimuthal angle $\varphi$ on the conversion of the incident plane wave is show in Fig.7-8. The curves presented are calculated at $\theta=80^{\circ}, \theta_{a}=45^{\circ}$ and $\varphi_{a}=0^{0}$. Note that in a uniaxial crystal the maximum angular separation of the " $\mathrm{o}$ " and " $\mathrm{e}$ " waves, in other words the maximum conversion of the incident linearly $s$ - or $p$ - polarized plane wave into the crosspolarized wave, occurs when the wave vector has the angle $\varphi_{\max } \approx 45^{0}$ with the optic axis [31]. Also we should note that the value of $\varphi_{\max }$ is proportional to $\left|n_{0}-n_{e}\right|$. Analyzing the figures 7-8, we can see that when the incident azimuthal angle $\varphi$ varies:

- the reflectance spectra demonstrate conversion of the incident s-polarized plane wave into the wave with same (Fig. 7) and orthogonal (Fig. 8) polarization. It occurs within the wide range of the incident azimuthal angles;

- the spectra also show a shift in the PBG spectral position when the azimuthal angle is changed. The largest shift, about $20 \mathrm{~nm}$, is observed under variation of the incident azimuthal angle $\varphi$ from $0^{0}$ to $90^{\circ}$. This results are in agreement with [39];

- the maximum conversion of the incident $s$-polarized wave into the wave with orthogonal polarization occurs at the incident azimuthal angle $\varphi \approx 45^{0}$ (see Fig. 8(b)), that is in conformity with [31]. In this case two reflection peaks with magnitude about 0.3 are clearly observed in the reflectance spectra $R_{p s}$. The first reflection peak with the central wavelength $780 \mathrm{~nm}$ 
is corresponding to the short-wavelength PBG edge for case $\varphi=90^{\circ}$. The second one with the central wavelength $835 \mathrm{~nm}$ - to the long-wavelength PBG edge for case $\varphi=0^{0}$

The influence of the sloping and the azimuthal angles $\theta_{a^{\prime}} \varphi_{a}$ (which determine direction of the optical axes) on the conversion incident $s$-polarized plane wave into wave with orthogonal polarization at the Bragg wavelength $\left(\lambda_{c}=800 \mathrm{~nm}\right)$ is shown in Fig. 9(a,b). Reflection coefficients $R_{p s}$ is calculated at incident angles $\theta=80^{\circ}$ and $\varphi=0^{0}$. It is clear from this graph that:

- the maximum value of $R_{p s}$ is around 0.4 (see Fig. 9(b)) and corresponds to the sloping angle $\theta_{a}=0^{0}$ and the azimuthal angles $\varphi_{a}=m \cdot \pi / 4,(m=1,3,5,7)$ that is in accordance with [31];

- in the case $\varphi_{a}=m \cdot \pi / 2,(m=0,1,2,3,4)$ the conversion of the incident wave into the wave with orthogonal polarizations is virtually absent $R_{p s} \approx 0$.

Finally, we can conclude that reflection coefficients of the investigated structures are very sensitive to the azimuthal angle $\varphi_{a}$ and the incidence angles $\theta$ and $\varphi$. When the angles of incidence $\theta=90^{\circ} \varphi=0^{\circ}$ and the azimuthal angle $\varphi_{a}$ possesses values $0^{\circ}$ or $90^{\circ}$, the $s$ - and $p$-modes are almost uncoupled, and the value of the $R_{p s}$ remains very small. The situation is similar to that one in the case of isotropic materials. With the exception of these particular values of $\varphi_{a^{\prime}}$ there is a great mixing between $s$-and $p$-modes that gives rise to the emergence of a large reflection coefficient $R_{p s}$.

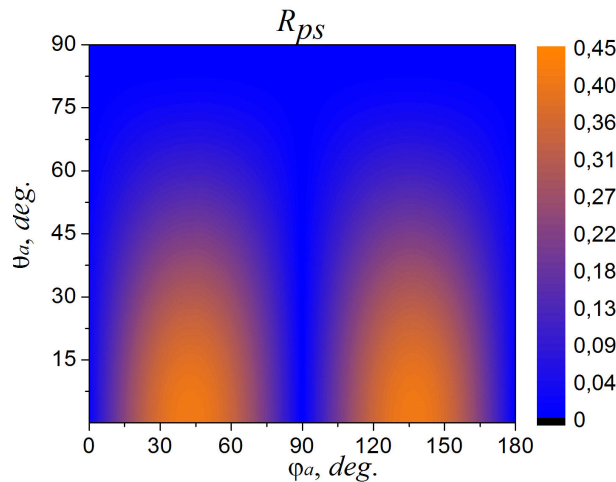

(a)

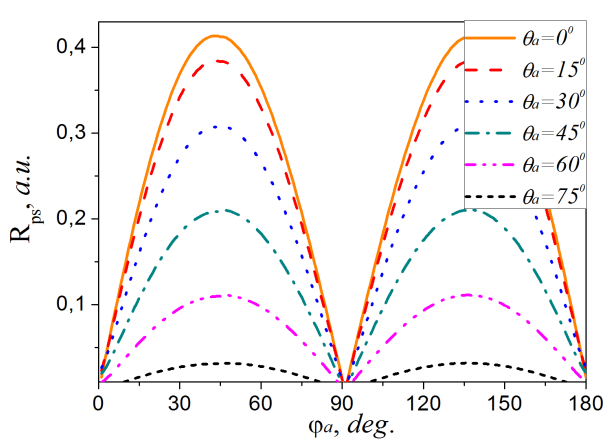

(b)

Figure 9. Reflection coefficient $R_{p s}$ as function of the sloping and the azimuthal angles

The PSi-based mirrors studied above are periodic structures, but one important property of $\mathrm{PhC}$ is the presence of narrow resonance (localized defect modes) in the PBG region when a disorder is introduced in their periodic structure. Usually "defect" is a layer with halfwavelength optical thickness that inserted in the middle of the dielectric stack. Fig. 10 shows 
a scheme of a typical PSi-based microcavity structure consisting in an active layer sandwiched between two distributed Bragg reflectors (PSi mirrors). At that, the reflectance spectrum of the whole structure is changed. As a rule, when the parameters of defect layer are properly selected, sharp transmittance peak appears within the main reflectance band. The wavelength of the transmittance peak corresponds to the resonant wavelength of the defect. For instance, if the thickness of the defect layer is twice larger than it was in defectless structure, the transmittance peak appears at the Bragg wavelength of the corresponding defectless structure. Thus, if the radiation with wavelength equal to Bragg wavelength of defectless reflector falls at this structure it will pass the structure almost without the reflectance. If the defect thickness will be slightly different, the wavelength of the transmittance peak will be different as well.

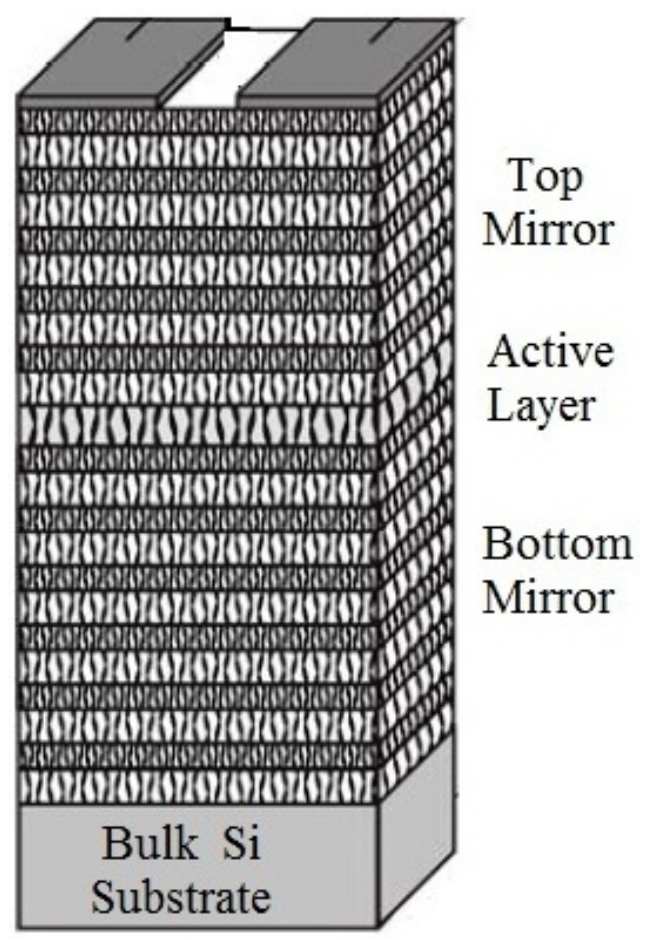

Figure 10. Schematic presentation of PSi-based microcavity

The advantage of using PSi microcavities is that the position of the transmittance peak is completely tunable by changing the properties of the central layer (i.e. porosity and thickness) during the electrochemical etching condition and by the infiltration of organic molecules (for example liquid crystals [32]).

Now, let's discuss the influence of anisotropy of layers on the optical properties of the microcavitiy. In cited case the PSi-based microcavity consist of two mirrors (each of them 
consists of 12 building blocks) separated by active layer with optical thickness $\lambda_{c} / 2$ and low porosity.

The Fig. 11 shows the reflectance spectra of the microcavity for both $s$ - (solid curve) and $p$ (dashed curve) polarized incident plane waves. It should be noted that spectra reveal the presence of photonic band gap with a reflectance of about 0.85-0.9 and microcavity mode within the PBG for both polarizations. Analyzing both spectra together, we can observe a spectral shift of the microcavity mode when the incident polarization is changed. This particular effect is also the manifests an attribute of birefringence and can be used in dividing the incident radiation polarization. In our case, the central wavelengths of resonance peaks are $\lambda_{s c}=796 \mathrm{~nm}$ and $\lambda_{p c}=817 \mathrm{~nm}$ for $\mathrm{s}$ - and $p$ - polarization, respectively. Taking into account this result, we can consider that the variation in the incident radiation polarization brings about the shift $\Delta \lambda=\left|\lambda_{p c}-\lambda_{s c}\right|$ of the microcavity mode, and this shift may be as high as $21 \mathrm{~nm}$ for presented case.

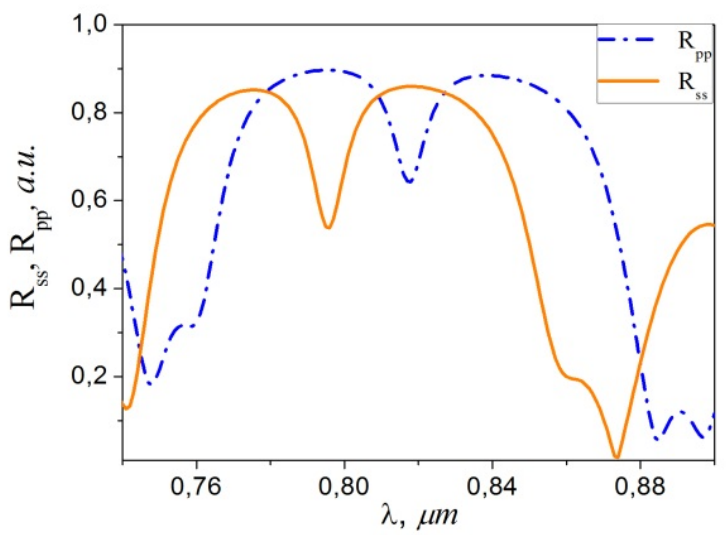

Figure 11. $\mathrm{s}$ - and $p$ - polarized reflectance spectra of microcavity. In this case $\lambda_{c}=800 \mathrm{~nm}, \theta=80^{\circ}, \varphi=\varphi_{a}=0^{\circ}, \theta_{a}=45^{\circ}$

Figure 12 plots the spectral shift of the microcavity mode for $p$-and s-polarized light, as a function of the of azimuthal angle $\varphi_{a}$. From this figure, some particular effects can be highlighted:

- the orientation of the optical axes of the layers with respect to incidence plane of $s$ - or $p$ polarized waves influences on the quantity of resonant peaks within PBG area and its location on the wavelength scale. Particularly, with the increasing of azimuthal angle, the "additional" well recognized resonant peak is appeared in the stopbands for both polarizations of the incident plane waves. It correlates with results of the paper [40]; 
- as a general trend, the value of the "main" resonant peak is decreasing with increasing the azimuthal angle $\varphi_{a}$ and it completely disappears when $\varphi_{a}=90^{\circ}$. There against, the magnitude of the "additional" resonance peak is increasing with increasing $\varphi_{a}$ and it has maximum value in the mentioned above case;

- both transmission peaks have identical magnitudes when azimuthal angle $\varphi_{a} \approx 48^{0}$ for both $s$ - and $p$-polarization;

- the extreme cases, namely $\varphi_{a}=0^{0}$ and $\varphi_{a}=90^{\circ}$, correspond to presence of single-defect mode within PBG region, while in the other cases this mode splits into two defect modes. The comparison of the results presented in Fig. 12(a) and 12(b) shows that $\lambda_{s 0}=\lambda_{p 90}=796 \mathrm{~nm}$ and $\lambda_{s 90}=\lambda_{p 0}=817 \mathrm{~nm}$. Here we used next definition for central wavelengths $-\lambda_{x y}$. In this notation, the left subscript index $x=s, p$ is corresponding to polarization of incident wave; the right subscript index $y=0,90$ is corresponding to quantity of azimuthal angle $\varphi_{a}$;

- also, in the extreme cases, we observed the shift of the resonance peaks $\Delta \lambda=\left|\lambda_{p 0}-\lambda_{p 90}\right|=\left|\lambda_{s 0}-\lambda_{s 90}\right|$ about $21 \mathrm{~nm}$ to the short-wavelength and long-wavelength regions for $p$ - and s-polarization, respectively. This result is in good agreement with the experimental results [39].

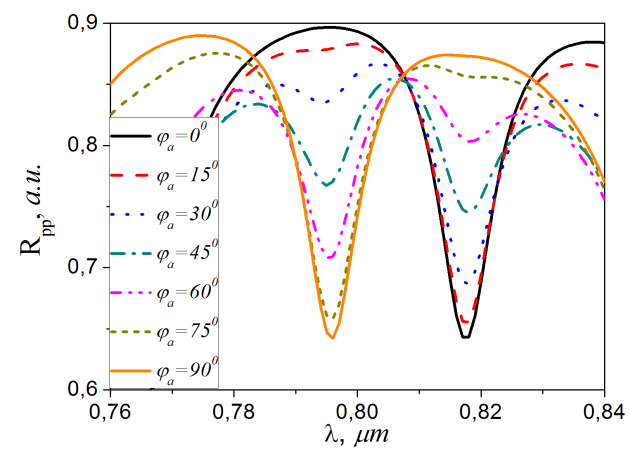

(a)

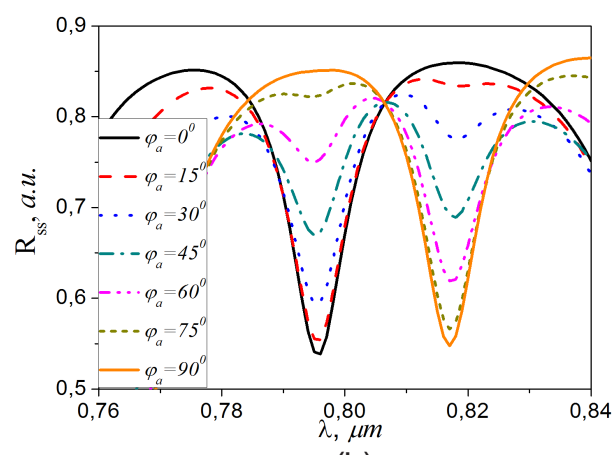

(b)

Figure 12. Microcavity resonance shift based on the azimuthal angle $\varphi_{a}$ change. Reflectance spectra for $p$ - and $s$ - polarized incident plane wave are depicted on Fig. 12(a) and Fig. 12(b), respectively.

The Fig. 13 shows characteristics similar to those one presented on Fig.12, but for crosspolarized components $R_{s p}$ and $R_{p s}$. In these figures we can see that within the PBGs areas the reflection coefficients $R_{s p}$ and $R_{p s}$ have local minimum that occur around the wavelength $\lambda \approx\left(\lambda_{p 0}+\lambda_{p 90}\right) / 2 \approx\left(\lambda_{s 0}+\lambda_{s 90}\right) / 2$. 


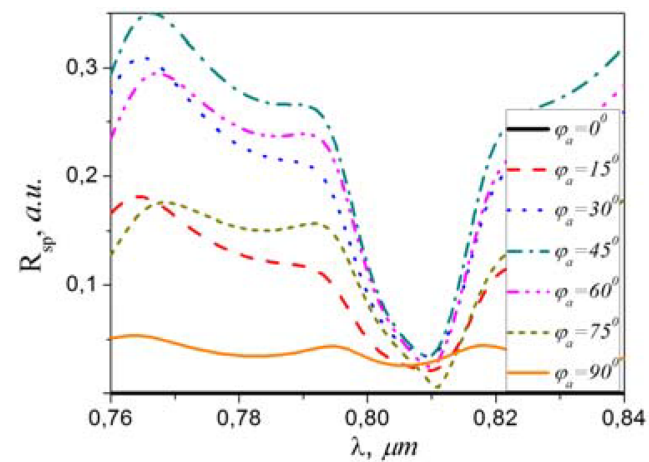

(a)

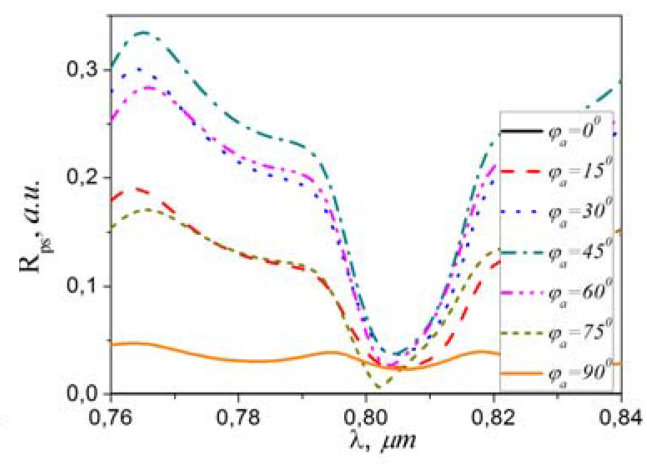

(b)

Figure 13. Reflectance spectra $R_{s p}(\mathrm{a})$ and $R_{p s}$ (b) as function of the azimuthal angle $\varphi_{a}$. In this case $\lambda_{c}=800 \mathrm{~nm}, \theta=80^{\circ}$, $\varphi=0^{0}, \theta_{a}=45^{0}$.

\section{Conclusions}

In conclusion, the present chapter describes the mathematical background for calculation of spectral characteristics of the birefringent layered media. On the basis of the presented theoretical description and its numerical approximation the influence of material anisotropy of PSi-based layered photonic structures on their optical properties has been theoretically investigated. All these multilayer PBG structures have been designed for $0.8 \mu \mathrm{m}$ applications.

The reflectance spectra of some photonic structures for both polarization of the incident plane wave are calculated. The agreement between the numerical calculations and the experiments [39] are obtained. It was shown numerically that anisotropy of layered media reduces to polarization transformation of the incident plane wave. Notably, the maximum conversion of incident plane wave into the wave with orthogonal polarization occurs when the optical axis of the structure have angles $\varphi_{a}=m \cdot \pi / 4,(m=1,3,5,7)$ with respect to the incidence plane of $s$ - or $p$-polarization incident plane. In contrast to this, the conversion of the incident wave into the wave with orthogonal polarizations is absent when $\varphi_{a}=m \cdot \pi / 2,(m=0,1,2,3,4)$.

Also from our numerical calculations, we conclude that the location of the PBG edges and location of microcavity modes within PBG region are different for $s$ - and $p$ - polarized waves and their spectral positions change under rotation of the optical axis of the structure with respect to the wave vector of the incident electromagnetic wave. The variation of polarization of the incident radiation brings about the shift of the microcavity mode, and this shift may be as high as $21 \mathrm{~nm}$ for discussed case. This particular effect can be used in the devices that divide the incident radiation according to polarization.

In addition the obtained results can be used in the designing of PSi-based photonic devices: 
- spectral- and polarization-selective elements (filters);

- electrically tunable filters and optical switches. In this instance it is required that the pore be filled with liquid crystals;

- polarization converters;

- time-delay elements for optically controlled phased-array antenna system.

\section{Author details}

V.I. Fesenko ${ }^{1}$, I.A. Sukhoivanov², S.N. Shul'ga ${ }^{3}$ and J.A. Andrade Lucio²

1 Institute of Radio Astronomy of National Academy of Sciences of Ukraine and Kharkov National University of Radio Electronics, Ukraine

2 University of Guanajuato, Mexico

3 Kharkov National University, Ukraine

\section{References}

[1] Yakushev S.O., Shulika O.V., Petrov S.I., Sukhoivanov I.A. Chirp Compression with Single Chirped Mirrors and its Assembly. Microelectronics Journal 2008; 39(3-4) 690-695.

[2] Iakushev S.O., Shulika O.V., Lysak V.V., Sukhoivanov I.A. Air-Gap Silicon Nitride Chirped Mirror for Few-Cycle Pulse Compression. Optoelectronics and Advanced Materials. Rapid Communications 2008; 2(11) 686-688.

[3] Guryev I.V., Shulika O.V.,Sukhoivanov I.A., Mashoshina O.V. Improvement of Characterization Accuracy of the Nonlinear Photonic Crystals Using Finite ElementsIterative Method. Applied Physics B - Lasers and Optics 2006; 84(1-2) 83-87.

[4] Klymenko M.V., Lysak V.V., Sukhoivanov I.A., Shulika A.V. Optical and Transport Properties of In0.49Ga0.51P/In0.49(Ga0.6Al0.4)0.51P Single Quantum Well Structure with Digital Alloy Barriers. Superlattices and Microstructures 2009; 46(4) 603-610.

[5] Klymenko M.V., Safonov I.M., Shulika O.V., Sukhoivanov I.A. Ballistic Transport in Semiconductor Superlattices with Non-Zero In-Plane Wave Vector. Phisica Status Solidi B 2008; 245(8) 1598-1603.

[6] Klymenko M.V., Safonov I.M., Shulika O.V., Sukhoivanov I.A., Michalzik R. EffectiveMass Superlattice as an Injector in Quantum Cascade Lasers. Optical and Quantum Electronics 2008; 40(2-4) 197-204. 
[7] Shulika O.V., Safonov I.M., Sukhoivanov I.A., Lysak V.V. Quantum Capture Area in Layered Quantum Well Structures. Microelectronics Journal 2005; 36, 350-355.

[8] Safonov I.M., Sukhoivanov I.A., Shulika O.V., Lysak V.V. Piecewise-Constant Approximation of the Potential Profile of Multiple Quantum Well Intrinsic Heterostructures. Superlattices and Microstructures 2008; 43(2) 120-131.

[9] Lysak V.V., Sukhoivanov I.A., Shulika O.V., Safonov I.M., Lee Y.T. Carrier Tunneling in Complex Asymmetrical Multiple Quantum Well Semiconductor Optical Amplifiers. IEEE Photonics Technology Letters 2006; 18(12) 1362-1364.

[10] Tuz, V., Prosvirnin S., Zhukovsky S.Polarization Switching and Nonreciprocity in Symmetric and Asymmetric Magnetophotonic Multilayers with Nonlinear Defect.Physical Review A 2012; 85 043822(1)-043822(8).

[11] Fesenko V.I. and Sukhoivanov I.A. Polarization Conversion in Inhomogeneous Anisotropic Multilayer Structures. In AIOM 2012: Lasers, Sources, and Related Photonic Devices, OSA Technical Digest (CD) (Optical Society of America, 2012), paper JTh2A.7, AIOM 2012, 1-3 February 2012, San-Diego, USA.

[12] Tuz V. Optical Properties of a Quasiperiodic Generalized Fibonacci Structure of Chiral and Material Layers. Journal of the Optical Society of America B 2009: 26(4) 627-632.

[13] Nefedov I.S., Tretyakov S.A. Photonic Band Gap Structure Containing Metamaterial with Negative Permittivity and Permeability Physical Review E 2002; 66(3) 036611(1)-036611(5).

[14] Pereira M., Shulika O. (Eds) Terahertz and Mid Infrared Radiation. Generation, Detection and Applications. NATO Series B: Physics and Biophysics. Springer; 2011.

[15] Sukhoivanov I.A., Guryev I.V. Photonic Crystals Physics and Practical Modeling. Springer; 2009.

[16] Dal Negro L., Boriskina S.V. Deterministic Aperiodic Nanostructures for Photonics and Plasmonics Applications. Laser Photonics Review 2011; 1-41.

[17] Yakushev S.O., Sukhoivanov I.A., Shulika O.V., Lysak V.V., Petrov S.I.Modeling and Simulation of Interaction of the Ultrashort Laser Pulse with Chirped Mirror for Structure Design Improvement. Journal of Optoelectronics and Advanced materials 2007; 9(8) 2384-2390.

[18] John S. Localization of Light in Periodic and Disordered Dielectrics. In: Confined Electrons and Photons, Plenum Press; 1995.

[19] Iakushev S.O., Sukhoivanov I.A., Shulika O.V., Andrade-Lucio J.A., Perez A.G. Nonlinear Pulse Reshaping in Optical Fibers. In: Laser Systems for Applications, InTech; 2011.

[20] Iakushev S.O., Shulika O.V., Sukhoivanov I.A. Passive Nonlinear Reshaping Towards Parabolic Pulses in the Steady-State Regime in Optical Fibers. Optics Communication 2012; 285(21-22) 4493-4499. 
[21] Corral J.L., Marti J., Fuster J.M. and Laming R.I. True Time-Delay Scheme for Feeding Optically Controlled Phased-Array Antennas Using Chirped-Fiber Gratings. IEEE Photonics Technology Letters 1997; 9(11) 1529-1531.

[22] Acoleyen K.V., Rogier H., Baets R. Two-Dimensional Optical Phased Array Antenna on Silicon-on-Insulator. Optics Express 2010; 18(13) 13655-13660.

[23] Komarevskiy N, Braginsky L, Shklover V, Hafner C, Lawson J. Fast Numerical Methods for the Design of Layered Photonic Structures with Rough Interfaces. Optic Express 2011; 19(6) 5489-5499.

[24] Mathew Ninan, Jiang Zhengyi, Wei Dongbin. Analysis of Multi-Layer Sandwich Structures by Finite Element Method. Advanced Science Letters 2011; 4(8-10) 3243-3248.

[25] Carretero L., Perez-Molina M., Acebal P., Blaya S., Fimia A. Matrix Method for the Study of Wave Propagation in One-Dimensional General Media. Optics Express 2006; 14(23) 11385-11391.

[26] Yeh P. Electromagnetic Propagation in Birefringent Layered Media. Journal of the Optical Society of America 1979; 69(5) 742-756.

[27] Taflove A., Hagness S.C. Computational Electrodynamics: The Finite-Difference TimeDomain Method, 2nd ed. Artech House; 2000.

[28] Thylen L. and Yevick D. Beam Propagation Method in Anisotropic media. Applied Optics 1982; 21(15) 2751-2754.

[29] Felsen L., Marcuvitz N. Radiation and Scattering of Waves. Wiley; 2003.

[30] Zhuck N.P. Electromagnetic theory of arbitrarily anisotropic layered media Part 1. Scalarization of field quantities. International Journal of Electronics 1993, 75(1) 141-148.

[31] Yariv A., Yeh P. Optical Waves in Crystals. Wiley; 1984.

[32] Tkachenko, G.V., Shulika, O.V. Thermal Tuning of a Thin-Film Optical Filter Based on Porous Silicon and Liquid Crystal. Ukrainian Journal of Physical Optics2010; 11(4) 260-268.

[33] Samuoliene N., Satkovskis E. Reflectivity Modeling of All-Porous-Silicon Distributed Bragg Reflectors and Fabry-Perot Microcavities. Nonlinear Analysis: Modeling and Control 2005; 10(1) 83 - 91.

[34] Charrier J., Le Gorju E., Haji L., Guendouz M. Optical Waveguides Fabricated from Oxidised Porous Silicon. Journal of Porous Materials 2000; 7(1-3) 243-246.

[35] HadjersiT., GabouzeN. Photodetectors Based on Porous Silicon Produced by AgAssisted Electroless Etching. Optical Materials 2008; 30(6) 865-869.

[36] Saarinen J., Weiss S., Fauchet P., Sipe J.E. Optical Sensor Based on Resonant Porous Silicon Structures. Optics Express 2005; 13(10) 3754-3764. 
[37] Pap A.E., Kordas K., Vahakangas J., Uusimaki A., Leppavuori S., Pilon L., Szatmari S. Optical Properties of Porous Silicon. Part III: Comparison of Experimental and Theoretical Results. Optical Materials 2006; 28 506-513.

[38] Kovalev D., Polisski G., Diener J., Heckler H., Künzner N., Timoshenko V.Yu., Koch F. Strong In-Plane Birefringence of Spatially Nanostructured Silicon. Applied Physics Letters 2001; 78, 916.

[39] Aktsipetrov O.A., Dolgova T.V., Soboleva I.V., Fedyanin A.A. Anisotropic Photonic Crystals and Microcavities Based on Mesoporous Silicon. Physics of the Solid State 2005; 47, 156-158.

[40] Ouchani N., Bria D., Djafari-Rouhani B., Nougaoui A. Defect Modes in One-Dimensional Anisotropic Photonic Crystal. Journal of Applied Physics 2009; 106 113107(1)-113107(8). 
